\title{
Navigating moments of uncertainty and socio-emotional risks in small-group work
}

\author{
Muxin Zhang and Katherine Ansell \\ Physics Department, University of Illinois Urbana-Champaign, 1110 W. Green St, Urbana, IL, 61801 \\ Eric Kuo \\ Departments of Physics and Curriculum \& Instruction, \\ University of Illinois Urbana-Champaign, 1110 W. Green St, Urbana, IL, 61801
}

\begin{abstract}
Supporting students' sense-making in discussions and labs is an important goal of college physics instruction. In this study, we explore how students navigate the socio-emotional risks of collaboration during moments of uncertainty while doing a lab activity. Attending to sense-making in the video recording of one group's work, we contrasted two episodes where the quality of their collaboration played out differently. We found that one of the group members changed how they navigated the tension that arose within the group from episode 1 to 2, going from disengagement to forceful idea-sharing, which increased the risk of disagreement in the second episode and hindered the group's sense-making progress. This case study shows how different approaches to socio-emotional risks could lead to different conceptual results of group work, contributing another example to a body of work showing how achieving our objectives for collaborative learning depends on careful attention to students' epistemological, conceptual, and socio-emotional resources.
\end{abstract}




\section{INTRODUCTION}

Collaborative learning has become an essential component of college-level science and engineering education due to its many benefits [1]. Studies found that, compared to students in individual learning conditions, students working in groups develop more sophisticated problem-solving skills [2], show more positive attitudes towards the subject [3], and achieve higher learning gains [4]. However, simply asking students to work in groups does not always lead to productive or meaningful discussions [5]. Factors such as perception of group dynamics can significantly impact the experience and result of collaboration [6]. Since collaborative learning in science hinges on complex processes such as building shared knowledge [7] and collaborative sense-making [8], successful group work depends on not only cognitive effort, but also epistemological framing [9], relational work [10, 11], and emotional engagement [12]. How these different dimensions come together during group work and how instructors can better support group work is an ongoing area of research.

We explore how the social and emotional dimensions of group work are entangled with students' sense-making during an introductory physics lab section. With video recordings of group work, we especially focus on moments when students experience uncertainty and socio-emotional risks concurrently, such as moments of confusion accompanied by heated disagreement between group members. Through indepth video analysis, we examine how a group of four students navigate two separate moments of uncertainty and how reactions to socio-emotional risks open up or close off opportunities for sense-making and consensus building. This study contributes to a vein of research investigating how socioemotional factors impact the substance and form of group discussions in physics.

\section{THEORETICAL BACKGROUND}

This study is situated within the context of college-level reformed physics labs [13], which are designed to create spaces for students to engage in sense-making and decisionmaking activities collaboratively, including generating hypotheses, examining assumptions, improving experimental designs, and troubleshooting equipment. During these activities, students often experience moments of uncertainty, such as feeling unsure about an idea or having doubts about what steps to take next. Although challenging to encounter, these moments are a crucial part of doing science and how students engage with science in classrooms [14, 15]. They are often the starting point of productive scientific argumentation [16], prompting students to articulate confusions and identify unresolved problems [17].

In group work, moments of uncertainty are not only cognitively challenging, but also socio-emotionally risky, involving potential relational conflicts [18] or potential embarrassment from not having the right answer [19]. These risks can drive students to stay silent when they are unsure and avoid ambiguities or contentions instead of engaging in deep sense-making. Researchers found that group mem- bers who work well together experience greater psychological safety, a shared sense that it is okay to take interpersonal risks [20]. Case studies of students working on physics problems showed that, when emotional tension arose within a group, students chose to shut down their conversation before they reached conceptual resolution in order to maintain social cohesion [21]. Some students also use discourse moves to create distance between their ideas and themselves to avoid potential embarrassment or frustration, creating a safer space for the group to sense-make [22].

Informed by these theories, we identify moments of uncertainty accompanied by socio-emotional risks as one of the key struggles that students experience during collaborative sensemaking, and we especially focus on these moments while analyzing videos of group work. This is a slightly different approach from studies exploring the interpersonal dimension of group work by identifying the kinds of social interactions that correlate with success in collaboration [4, 23, 24]. While these studies help instructors recognize the value of certain kinds of interactions, the question of why some students engage (or don't engage) in high-quality interactions remains open. By centering the key struggles of group work in this study, we intend to make sense of students' diverse collaborative approaches and behaviors during difficult moments.

Our two research questions are: 1) How do students navigate moments of uncertainty and socio-emotional risks during group work? and 2) How may these moments impact the group's sense-making and decision-making during a lab activity?

\section{METHODS}

We use a case study approach to analyze two episodes of collaboration. The two episodes we selected were from the same group of students discussing similar topics, yet they had different conceptual endings, acting as "maximum variation cases" [25]. By comparing them, we could identify what changed and what stayed consistent in how students approached group work, thereby gaining insights about what led to the socio-emotional and interactional differences between the two episodes.

For each case, we use an inductive thematic analysis approach [26] to identify patterns from thick, descriptive coding of video data without subscribing to any existing coding scheme. From these patterns, we generate theories in an abductive and informed manner, taking into account existing theories of cognitive, epistemological, and socio-emotional dimensions of collaborative learning while staying grounded to the data [27].

\section{A. Study Context}

The data corpus for this study consists of video recordings from the first semester of an introductory calculusbased mechanics laboratory reform project using the Interactive Online Laboratory (IOLab) system, a multi-component measuring device paired with data analysis software that students use to perform physics experiments both at home 
and in classrooms [28]. This is a large-enrollment class at a large Midwestern university and the demographics are majority white and male. In a typical lab, students are given under-specified ("ill-structured") instructions that elicit sense-making throughout the experimental process [29].

We chose to focus on the sixth lab of the semester for this study, because the lab activity was specifically designed to challenge students' prior assumptions about tension forces. The lab prompt asked students to connect two IOLab devices by a string, leave one on the table, and let the other one hang over the edge of the table. Students were asked to test the hypothesis that, when the devices are released, the tensions measured on two ends of the string are equal to each other. When the forces are measured properly, students should find out that the tensions are significantly different from each other due to the frictional force on the string from the edge of the table.

We randomly selected groups to record during the semester, collecting the video using a GoPro camera attached to the ceiling and the audio with a voice recorder placed on the table. We watched several groups but chose one group to analyze in depth because we noticed several moments of disagreement and emotional tension in their video. The group that we focused on has four students sitting at a table, Alex, Blake, Cam, and Dani (pseudonyms). We perceive all four students as male based on their real names and appearances, but since we did not collect reliable self-reported gender data at the moment of recording, we will use they/them pronouns to refer to them in this paper.

\section{B. Analytic Process}

We started the analytic process by familiarizing ourselves with the video and generating content logs. Meanwhile, we marked moments in the video when uncertainty or socioemotional risks arose. Operationally, we defined "moments of uncertainty" as moments when group members have different ideas and there is no immediate consensus on next steps, and we defined "socio-emotional risks" as situations that drive group members to make moves to avoid open conflict or relational damage among themselves. We then selected two episodes, 4 minutes and 2 minutes long, that constituted the data set of this case study and transcribed both episodes. We identified these two episodes as cases of students encountering moments of uncertainty and socio-emotional risks concurrently during group work.

We present these two episodes as contrasting cases because they unfolded very differently in terms of how the group discusses ideas and determines what to do next. In the first episode, an initial disagreement is followed by a group member's withdrawal, subsequent group reasoning and agreement, and social repair. By contrast, in the second episode, disagreement led to one group member's idea not being fully considered, cutting short the group's sense-making before moving on.

For each episode, we generated in-depth descriptive accounts to capture what was happening reasoning-wise and socio-emotionally from moment to moment, using evidence such as students' discourse, tone, loudness, gestures, and postures to interpret their behaviors and emotional expressions. Reasoning descriptions included what key physics- and experiment-related ideas were being discussed, what the participants were disagreeing about, and what task-based decisions they made. Socio-emotional descriptions included what students did interpersonally and how they did it (e.g., introduced an idea with an uncertain tone of voice) including their social moves and emotional expressions. We also described certain components of the classroom context that were relevant to each moment, such as the physical positioning of the students and the laboratory equipment. We constantly went back and forth between watching the raw video footage and generating descriptive accounts to further confirm, refine, and enrich our descriptions.

To find potential patterns and meaningful themes in the dense descriptive accounts, we summarized how events unfolded on the group level and how each group member approached group work on the individual level. Then, we made systematic comparisons to answer our two research questions. First, to understand how students navigated the two moments of uncertainty, we attended to how three students approached the uncertainty differently and how their ways of interacting differed from the first and second episode, noticing shifts in their discussion of reasoning and socio-emotional group engagement. Second, to understand how their socioemotional navigation impacted the group's sense-making, we examined how the events unfolded chronologically and generated substantive theories to explain the result of their negotiation.

\section{FINDINGS}

\section{A. Episode 1: How to calibrate the force probes?}

In this episode, the group grappled with a disagreement about how to calibrate the IOLab force sensor when measuring the tension force on a string. This disagreement generated some emotional tension within the group, especially between Cam and Dani.

While the group was getting ready for their first lab activity, Alex commented on what they needed to measure. Following Alex's comment, Cam suggested a plan for how to zero the force sensor, to which Alex agreed, but Dani raised a concern related to the previous week's lab activity:

Cam: And we have to zero it when it's hanging off the edge, right?... That what we did last week.

Alex: Yeah.

Dani: Wasn't it an issue last time we would zero when it's hanging down and we had to constantly re-zero it when it was on the table?

Cam: Umm...

Dani: Do you remember? You always had me pull it up...

Cam: Yeah. But we... cause we had to, right?

Dani: But why wouldn't we have to do it this time?

Cam: We still have to have the same type of measurement... we have to have it zero when it's hanging off the edge... We are trying to measure the tension... so we want it 
when it starts moving.

Dani: But that includes the tension, when it's hanging...

Alex: Let's just... do it, and see what happens (At this point Dani leans back in their chair and makes a "hands-off" gesture.)

Cam: Yeah, yeah. I like that idea. If we run into a problem, we'll know and we'll fix it.

The main debate here was between Cam's idea of zeroing the force sensors when one probe is hanging off the edge of the table and Dani's point that they should zero the force sensors with both resting on the table. Dani introduced a series of challenging questions to Cam's ideas ("Wasn't it an issue last time...?", "Do you remember?" and "But why wouldn't we have to do it this time?" ), and Cam supported their original suggestion. During these turns, Cam and Dani were making eye contact with each other. The quick turn-taking and word choices like "have to" gave the conversation a tug-of-war feeling. Perhaps sensing growing tension, Alex jumped in to propose that they can move on with experiment for now and "see what happens," pointing a way for the group to move forward. Cam immediately agreed with Alex, but Dani moved back in their chair and put up their hands, signaling a disengagement from the conversation.

After this, Cam led the group to set up the experiment and get ready for their first trial. Cam controlled the laptop and zeroed the force probes with one hanging off of the table. During this time, Cam expressed some frustration with not being able to manipulate the data collection software smoothly, showing a sense of urgency in making progress. Dani helped out when called upon by Cam to catch the force probes at the end of their motion but did not offer any ideas or contributions during this process. Alex actively helped Cam, paid close attention to the data collected on the laptop screen, and made a few comments to make sense of the data. After one data collection trial, Alex raised an issue, which was in line with Dani's original suggestion:

Alex: I don't think... we need... No we need to re-zero, rezero it when it's on the table... Because when it's hanging off, the force up should not be zero.

Cam: Oh, right.

Dani: That's what I said.

Cam: Okay, I'm sorry. My bad. I know.

Alex: It's been a week. (smiles)

Blake: It's only like... 8 am... partially functioning.

Cam: Alright. It's zeroed. You can hang it off the edge now.

We argue that much of what occurs in this episode emerges from how individuals react to potential socio-emotional risks in the group. When tension arose between Cam and Dani's disagreement, Alex jumped in to propose delaying the argument, attempting to relieve that tension. After that, Dani disengaged from the discussion. We can interpret Dani's disengagement as the result of personal frustration that Dani felt at not having their idea heard initially. When Dani said "That's what I said" at the end, it further communicates Dani's frustration that this idea was not taken up when they suggested it. In their responses, the rest of the group attended to the socio- emotional implications of Dani's comment. Cam's apology attended to Dani's frustration and accepted blame for not hearing Dani's original idea. Alex and Blake's comments smoothed over the tension within the group, attributing this event not to Cam, but to other external factors that explain the miscommunication: a rough week and early class time.

We also see in this episode that students' socio-emotional navigation impacted the group's progress in thinking about the uncertainty they encountered during data collection. Although Cam and Dani's debate at the start of this episode surfaced a key issue about setting up the lab, Alex's proposal and Dani's disengagement delayed the resolution of this issue. At the same time, this delay allowed the group to move forward and try out Cam's data collection suggestion. In the process, Alex was able to present a new articulation for Dani's original idea that convinced Cam. Although perhaps not collaboratively ideal, the group members' socio-emotional negotiations, particularly Alex's and Dani's moves, actually allowed the group space to make progress and eventually come to agree with Dani's original suggestion with satisfying justifications.

\section{B. Episode 2: Should the probes read the same initially?}

This episode immediately followed the first episode. After zeroing both force sensors on the table, the group hung one of the sensors off the table and held the system at rest. At this point, they found that the forces measured by the probes were not equal. This led to a debate as to whether the tensions should be equal when the sensors are at rest and whether this was the empirical question being tested in the lab.

Cam started the discussion by raising a problem with the data they were seeing on their laptop, saying that the force probes did not read the same initially, claiming that they should. Alex responded to Cam's comment with doubt, saying that the probes did not have to read the same at the beginning, and Dani disagreed with Alex, saying that the force probes should read the same. Their contention escalated quickly:

Cam: Except, that probe is not correct.

Alex: Which one?

Cam: The second... This one.

Alex: They both should not be zero, because this is pulling

Cam: Yeah. But they should both be negative two.

Alex: Not really...

Dani: Yeah it should be. The tension is exactly the weight...

Cam: (touching the force probe) There we go. There. There I got it now.

Dani: ... when they are... at rest.

Alex: You bent it. That's cheating. (points at Cam) (pauses) No. We're tr.. we are testing to see it IF they would be the same.

Dani: They would have to be the same at rest. We are testing if they are the same when it's moving. When they're at rest, they HAVE to be the force of weight.

Cam: Okay. There. We're good. (Looking at laptop screen)

Dani: Yes. I guarantee you. 
Alex: That makes sense. That makes sense. (pauses) Do you wanna try re-zeroing it again? Pull that up and re-zero both of them?

Cam: No... It's fine... Ready?

During the contention, Cam bent the force probe to "fix" the problem. When Alex continued to disagree with Cam and Dani, Dani stated their ideas assertively: "They would have to be the same at rest... When they're at rest, they have to be the force of weight," speaking with higher volume and putting extra emphasis on "have to." Alex immediately stepped back from arguing and threw their hands up, to which Dani added "I guarantee you." Alex responded "That makes sense. That makes sense." with visible discomfort. Seeing that Cam had bent the force probe, Alex suggested to Cam to re-zero the force probe, but that suggestion was dismissed by Cam.

Attention to socio-emotional dynamics again helps us understand the group's activity in this episode. Rather than wording their challenges as questions like they did in episode 1, Dani phrased them as statements, including definitive phrases like, "they have to be." and "I guarantee you." This shift is perhaps a reaction to Dani not having have their idea taken up when phrased as a question in the first episode. Along with this shift in phrasing, Dani's tone was forceful, perhaps attempting to prevent further challenges that may derail the group's progress. Along the same lines: why does Alex back off from their initial concern? Although Alex clearly was not convinced by Dani's argument, indicated by their suggestion to re-zero the probes and make the measurement again, Dani's assertively stated counters increased the socio-emotional risks of disagreeing with them at the moment, leading Alex to step back with a concessionary "That makes sense."

We see again that these socio-emotional dynamics have consequences for how the group proceeds with the lab. Unlike in episode 1, the group did not get to delay and open up space for sense-making, discussion, and eventual consensus. Dani's statements, Alex's concession, and Cam's control of the equipment, led the group to move forward without making sense of the uncertainty or resolving Alex's concerns.

\section{Summary of Findings}

In both episodes, members of the group expressed multiple ideas about how to proceed in their lab assignment and discuss these ideas. In both episodes, the discussion was happening in parallel with experimental actions, primarily led by Cam, who was in control of the equipment and continued to push the group's lab activity forward. However, in episode 1, the vocal members of the group came to consensus on how to proceed, whereas Alex's idea was never fully considered during, or even after, episode 2.

By contrasting these two episodes, we highlight how changes in socio-emotional navigation impact whether the group makes space to discuss uncertainty and resolve disagreements. In episode 1, Alex's delaying move and Dani's disengagement created space for the group to discuss how the force probes should be calibrated. In episode 2,
Dani's assertive statements-plausibly linked to their previous frustration-increased the socio-emotional risk of disagreeing with them in-the-moment, leading Alex to back down from their idea and allowing the group to move on without addressing Alex's concern. In this way, the outcomes of these disagreements were not purely based on students' physics knowledge or argumentation skills, but also on how the group members navigated the socio-emotional tensions that arise from their disagreements.

\section{DISCUSSION}

Collaborative small-group learning environments, like physics lab courses, are often designed with the intent that students can think through arguments, generate ideas, and learn by talking together. This is only enhanced by PERbased lab reforms, where students are asked to reasoning with models [30], make decisions with agency [31], and explain the validity and uncertainty of measurements [32]. This case study provides an empirical example of how the quality of a group's discussion may rely not only on conceptual and epistemological factors, but also socio-emotional ones. The group we observed actively discussed key ideas and collaborated with each other during both episodes, yet they differed in terms of how well they provided room for students to disagree and think through ideas, due to shifts in socioemotional navigation within the group from moment to moment. Although one episode might have had a more desirable ending than the other, we do not mean to imply what the group members should or should not have done due to the complexity of their in-the-moment interactions. The theoretical lens we chose also has the limitation that we did not consider other potentially important factors, such as gender or race.

This case study adds another example to a body of research that explores the consequential role of social and emotional dynamics in physics group work, such as studies identifying strategies that students use to manage socio-emotional risks $[21,22]$ and studies that explore students' emotional response to moments of uncertainty $[15,33]$. However, in contrast to these previous studies, which show how socioemotional risks like frustration and group tension threaten productive group sensemaking, the group's response to socioemotional risk in episode 1 , intentionally or not, had the consequence of creating space for the group to sense-make and reach consensus. Future studies can explore this potentially novel socio-emotional dynamic, through analysis of similar cases, longitudinal analysis of multiple groups, and conducting interviews with students to further understand their socioemotional experience of group work.

\section{ACKNOWLEDGMENTS}

Data collection was supported by NSF TUES Grant No. 1122534. We want to thank Mats Selen for co-creating the lab content, and Stina Krist for her feedback during data analysis. 
[1] L. Springer, M. E. Stanne, and S. S. Donovan, Effects of SmallGroup Learning on Undergraduates in Science, Mathematics, Engineering, and Technology: A Meta-Analysis, Review of Educational Research 69, 21 (1999).

[2] P. Heller, R. Keith, and S. Anderson, Teaching problem solving through cooperative grouping. Part 1: Group versus individual problem solving, American Journal of Physics 60, 627 (1992).

[3] I. A. Shibley and D. M. Zimmaro, The Influence of Collaborative Learning on Student Attitudes and Performance in an Introductory Chemistry Laboratory, Journal of Chemical Education 79, 745 (2002)

[4] M. Menekse and M. T. H. Chi, The role of collaborative interactions versus individual construction on students' learning of engineering concepts, European Journal of Engineering Education 44, 702 (2019).

[5] M. Summers and S. Volet, Group work does not necessarily equal collaborative learning: evidence from observations and self-reports, European Journal of Psychology of Education 25, 473 (2010).

[6] E. J. Theobald, S. L. Eddy, D. Z. Grunspan, B. L. Wiggins, and A. J. Crowe, Student perception of group dynamics predicts individual performance: Comfort and equity matter, PLOS ONE 12, e0181336 (2017).

[7] J. Roschelle and S. D. Teasley, The Construction of Shared Knowledge in Collaborative Problem Solving, in Computer Supported Collaborative Learning, NATO ASI Series, edited by C. O’Malley (Springer, Berlin, Heidelberg, 1995) pp. 6997.

[8] T. O. B. Odden and R. S. Russ, Defining sensemaking: Bringing clarity to a fragmented theoretical construct, Science Education 103, 187 (2019).

[9] R. E. Scherr and D. Hammer, Student Behavior and Epistemological Framing: Examples from Collaborative ActiveLearning Activities in Physics, Cognition and Instruction 27, 147 (2009).

[10] N. Miyake and P. A. Kirschner, The Social and Interactive Dimensions of Collaborative Learning, in The Cambridge Handbook of the Learning Sciences, Cambridge Handbooks in Psychology, edited by R. K. Sawyer (Cambridge University Press, Cambridge, 2014) 2nd ed., pp. 418-438.

[11] B. Barron, When Smart Groups Fail, Journal of the Learning Sciences 12, 307 (2003).

[12] C. Polo, K. Lund, C. Plantin, and G. P. Niccolai, Group emotions: the social and cognitive functions of emotions in argumentation, International Journal of Computer-Supported Collaborative Learning 11, 123 (2016).

[13] E. Etkina and A. Van Heuvelen, Investigative Science Learning Environment - A Science Process Approach to Learning Physics.

[14] S. A. Kirch, Identifying and resolving uncertainty as a mediated action in science: A comparative analysis of the cultural tools used by scientists and elementary science students at work, Science Education 94, 308 (2010).

[15] J. Watkins, D. Hammer, J. Radoff, L. Z. Jaber, and A. M. Phillips, Positioning as not-understanding: The value of showing uncertainty for engaging in science, Journal of Research in
Science Teaching 55, 573 (2018).

[16] Y.-C. Chen, M. J. Benus, and J. Hernandez, Managing uncertainty in scientific argumentation, Science Education 103, 1235 (2019).

[17] A. M. Phillips, J. Watkins, and D. Hammer, Problematizing as a scientific endeavor, Physical Review Physics Education Research 13, 020107 (2017).

[18] C. De Dreu and A. Van Vianen, Managing relationship conflict and the effectiveness of organizational teams, Journal of Organizational Behavior 22, 309 (2001).

[19] E. Goffman, Embarrassment and Social Organization, American Journal of Sociology 62, 264 (1956).

[20] A. Edmondson, Psychological Safety and Learning Behavior in Work Teams, Administrative Science Quarterly 44, 350 (1999).

[21] E. R. Sohr, A. Gupta, and A. Elby, Taking an escape hatch: Managing tension in group discourse, Science Education 102, 883 (2018).

[22] L. D. Conlin and R. E. Scherr, Making Space to Sensemake: Epistemic Distancing in Small Group Physics Discussions, Cognition and Instruction 36, 396 (2018).

[23] S. Shehab and E. Mercier, Exploring the Relationship Between the Types of Interactions and Progress on a Task during Collaborative Problem Solving, 14th International Conference of the Learning Sciences (ICLS) 3, 1285 (2020).

[24] D. T. Brookes, Y. Yang, and B. Nainabasti, Social positioning in small group interactions in an investigative science learning environment physics class, Physical Review Physics Education Research 17, 010103 (2021).

[25] B. Flyvbjerg, Five Misunderstandings About Case-Study Research, Qualitative Inquiry 12, 219 (2006).

[26] V. Braun and V. Clarke, Using thematic analysis in psychology, Qualitative Research in Psychology 3, 77 (2006).

[27] R. Thornberg, Informed Grounded Theory, Scandinavian Journal of Educational Research 56, 243 (2012).

[28] iOLab Solution for Physics Labs, Macmillan Learning for Instructors .

[29] K. Ansell and M. Selen, Student attitudes in a new hybrid design-based introductory physics laboratory, Physics Education Research Conference Proceedings, 36 (2016).

[30] D. R. Dounas-Frazer, K. L. Van De Bogart, M. R. Stetzer, and H. Lewandowski, Investigating the role of model-based reasoning while troubleshooting an electric circuit, Physical Review Physics Education Research 12, 010137 (2016).

[31] N. Holmes, B. Keep, and C. E. Wieman, Developing scientific decision making by structuring and supporting student agency, Physical Review Physics Education Research 16, 010109 (2020).

[32] D. Hu and B. M. Zwickl, Examining students' views about validity of experiments: From introductory to Ph.D. students, Physical Review Physics Education Research 14, 010121 (2018).

[33] J. Radoff, L. Z. Jaber, and D. Hammer, "It's Scary but It's Also Exciting": Evidence of Meta-Affective Learning in Science, Cognition and Instruction 37, 73 (2019). 